\title{
The qualitative approach toward education of environmental design
}

\author{
Somayyeh Bitaraf*, Reza Naghdbishi \\ Department of Architecture, Roudehen Branch, Islamic Azad University, Roudehen, Iran
}

Email address:

ar.s_bitaraf@yahoo.com (S. Bitaraf),reza.naghdbishi@riau.ac.ir (R. Naghdbishi)

\section{To cite this article:}

Somayyeh Bitaraf, Reza Naghdbishi. The Qualitative Approach toward Education of Environmental Design. Education Journal.Special Issue: Interdisciplinary Researches in Environmental Design Education. Vol. 4, No. 1-2, 2015, pp. 10-15. doi: 10.11648/j.edu.s.2015040102.12

\begin{abstract}
The subject of the present research is the analysis and evaluation of effects in way of separation, companionship, and bordering the private, semi- private and public areas within the collective settlements with focus on way of creating a livable setting. The field studies signify that the primary principles and fundamentals of architecture and urban setting landscape are employed at low level in most of the collective settlements and townships in Iran and the higher requirements such as sense of place, self-esteem, self-actualization, and or cognitive and aesthetic requirements have been ignored in design of them. The present investigation has proposed the educational strategies and some criteria for design of setting in collective settlements with respect to environmental psychology basic concepts like privacy, personal setting, territory, traffic, and paying attention to individuals' needs, wishes, and culture to achieve the preparation for more efficient and livable settings and areas. The above-mentioned study has been carried out by means of survey- descriptive research aiming at identifying cause-and-effect relation in order to describe the attributes of statistical population and way of nature under the existing conditions and the relationship between the events at small scale by adaption of cross- sectional and longitudinal techniques. Then, the hypotheses were explored by means of relational- correlation method along with abstract paradigms as environmental data through recognizing the public attitude toward the environmental by non- interventionist approach and the field studies were conducted through qualitative and quantitative methods. Eventually, this trend led to analysis and recording the results and proposing a strategy for conversion of design principles into educational trend of environmental design in this regard.
\end{abstract}

Keywords: Territorism, Collective Settlement, Environmental Perception, Environmental Planning, Behavior Setting, Education of Environmental Design, Livability

\section{Introduction}

Human is the product of environment, biology, and interaction among these two factors with each other and various physical factors may permanently affect on human. The human's presence and activity in several settings leads to creation, enhancement and continuance of environment. In addition to physical needs in paying human's attention to design some other requirements such as determination of territory, privacy, security, and social relations and or symbolic beauty are assumed as some of basic needs for human.

As the most private and foremost territory for human and a model for living setting for him/ her, the settlement emphasizes in this point that the members of community need to living in a secure, livable, and dynamic climate in which they can feel sense of comfort and belonging and it can be effective in improving the collectivistic spirit through creating various settings to pass some part of life collectively within the public and semi-public areas and in order to enhance the spirit of normal life. Paying attention to creating efficient areas in terms of architecture with considering the guidelines of environmental psychology as an integrated system is deemed as one of the requirements for design of townships and collective settlements.

After analysis and evaluation of the effects of separation, companionship, and proper bordering along the private and public areas in design of a livable climate in a collective settlement in this study, it is intended to propose a model by aiming at improvement of quality in areas of collective settlements whether private and or public as well as preparation of ground for the theory to employ it in education of environmental design. 


\section{Research Theoretical Framework}

\subsection{Definition of Livability}

\subsubsection{Terminology}

In the dictionary, term 'Livability' has been introduced as synonymous with words lively, liveliness, vitality, and vivacity [1]. Since 1981, this term has been denoted mainly as livability, liveliness, and quality of life in technical literature of urban design that shows the livability regarding life setting.

\subsection{Theories of Livability and Spaces}

In national charter of arts [2] it is argued that the livable community needs to an environment that facilitates potential for physical, spiritual, and intellectual growth for the individuals and provides sense of security, position, privacy, local society, and happiness and its scale to be coordinated with human's requirements and encourages for the balanced relationship among nature and environment and contributes to saving and conservation of consuming natural resources. If human's requirements are not considered in design the social environment will not be able to interact with them and consequently the audiences will not highly welcome it.

Bentley [3] emphasizes that the human-made environment may affect on possibility to enter and or not enter in those areas with improving the opportunities and with maximization of the range of presentable choices to the people. He assumes the influence, variation, legibility, flexibility, sensational richness, and sense of belonging as the effective factors in rising presence of users and improving the responsiveness level of environment.

Soltanzadeh [4] maintains that the livability includes two procedures where the first procedure is related to people's insight, culture, and perception and the other one is ascribed to urban and architectural spaces so that both of these two procedures are interrelated Considering these points may serve as a strong step taken toward creating more efficient climates with healthier relations where a spectrum of various experiences with happiness flows in other words one pole of this trend includes superficial and transient happiness while the other pole is deep and viable so that one could refer to concept of variety of activities in the public field and its compliance with the spaces within the framework of system of behavioral bases at micro level. Lack of relationship among design of elements and activities, which should be done, may undermine design [5]. Some of existential parameters of livable environments include rate of density of pedestrians, quantity, variation, and nature of behavioral bases, seasonal activities, rhythm and speed of urban setting, various users, form, and color are considered. Proposing a comprehensive definition from this concept requires considering the relations of this quality and other qualities in environment. Similarly, the way of arrangement in green space, decoration of similar building, the existing appropriate trees in spaces and routes, way of installation of electric lighting posts, telephone box, newspaper kiosk, transportation stations and more importantly maintenance of the effective traditional symbols may play essential role in improving social spirit for design and arrangement of areas within a collective settlement [6]. The importance of the abovementioned issues in residential area may seem more significant due to further relationship between more individuals to the extent that the works of well-known architects have also needed to several changes in spaces at the limited level and even at large scale by users in order to modify their own life setting in compliance with their own interests, desires, and requirements.

Lynch [7]assumes seven main axes for quality of an urban space i.e. livability, proportion, access, supervision, option, efficiency, and justice.

\subsection{Psychological Approach to Livability}

According to viewpoint of psychologists, there are various attitudes toward livability. These theories denote quality of life, wideness, expansion, hope, and desire originate from life experiences [8]. Quality of life is individual's perception from welfare that seems it is caused by satisfaction and or dissatisfaction with life in main areas [9]. Quality of life includes physical, psychological, and social dimensions, which are restricted to experiences, beliefs, expectations, and perception [10]. Likewise, it is also argued in World Health Organization (WHO) that quality of life is called to personal impression of a person from the position of his/ her life in relation to the given culture and value system in the community as well as its relation with his objectives, expectations, standards, and needs [11]. Despite of difference in opinions about definition of quality of life, there is an agreement in positive dimensions of life and including a multidimensional concept. The studies during recent decades suggest that through involvement of five senses in life environment by the aid of principal design and arrangement of spaces one could prepare the grounds for acquisition of enjoying presence in this setting and creating sense of livability all the times and under any condition.

In this course, livability can be explored from two attitudes:

- Environmental livability comprises of ecologic stability regarding variation and variability including acoustic noise and air pollution, energy loss, traffic mixing with green space and design attitudes such as changes and instability like legibility, sense of place, architectural distinctions, conditions and regulations in various points of city, quality of lighting posts and type of their safety and security and amiable and psychological proximity to urban setting.

- Cultural livability denotes survival, respect to an urban setting and its people that express identity, memory, tradition, social praising, delivery and distribution of consuming product and commodities and symbols explaining the distinctive nature of a urban environment [12].

\subsection{Role of Formal Elements of Space in Spatial Relations}

\subsubsection{Livability and Color}

The color is one of the paramount visual elements, which affects efficiently in public and private climates and one of its 
features is in that along with form, dimensions, type, and texture of them it identifies the volume and space of them for us. Colors possess perceptional weight and their arrangement together and their contextual and background role may also penetrate in these dimensions. Dark and incongruent spaces may cause the persons to suffer from unwanted stresses. The bright colors may lead to faster distinguishing of objects from the environment and improve the quality and quantity of their use. The colors may be seemed warmer, brighter, and shinier and create farsightedness in the person. The colder colors act inversely [13]. Yellow and purple are neutral namely they neither advance nor retreat. Similarly, the colors with little hue are used for more energetic environments and the stronger colors are employed for emphasis and stimulation. Grey is in harmony with any other colors. At the same time, one could unify the space by means of color and or make it distinct and recognizable. Age of users is also an important factor. Generally, the warm colors are deemed important in early years of life while cold colors are deemed crucially significant during puberty period.

\subsubsection{Complementary Contrast}

We call colors contrast as complementary when the pigments create the neutral color of grey- black by mixing. They are opposite but request for each other and in the case of lack of above color in eye, it is created automatically, these pairs of colors are (yellow- violet), (blue- orange), and (redgreen) [14]. By considering the spatial features and with emphasis on psychological attitude to it, using the color may have key effect on human's perception. Along with light, the colors create different background of contrasts, especially in line of demarcation among spaces during several hours a day and they make space various and livable. Such features in space may stimulate vision and tactile senses in such a way to become compatible with spatial function and idea for design.

\subsubsection{Impact of Statue on Spaces}

Using element or statue in a space may create variation, livability, legibility, training of mind, beauty, and improving visual quality [15] and add a new dimension to the given space. One of elements, which are responsible for identity and livability and spatial organization, is furniture. A wide group of tools, symbol, micro building, space, and elements has been typically improved and they emerge in novel and livable form.

\subsection{Elements of Livability}

Based on the resulting analyses from theories of livability, the elements of livability can be divided into four groups:

- Performance elements: They include variation of activities, flexibility, and quality of environmental security for activities.

- Aesthetic- empirical elements: Visual influence, various forms legibility, visual proportion, sensational richness, attachment, and security.

- Ecologic elements: Including quality of microclimates of space (climatic comfort) such as sunlight radiation, existing shade, wind, moisture, shadow, quality of smell, fragrance, and sounds in environment as well as reduced air pollution, land, and water

- Penetration element: This element is particularly important both at functional level and aesthetic level.

\subsubsection{Physical Penetration}

It means those spaces, which give opportunity to people to choose, should be accessible so that they can go from a place to other location in them [13]. As a stimulant factor, creating continuous and permeable (influential) space in public and private areas can be effective in improving relations among the persons. The formal and visual penetration depends on design of access network and dividing the environment. Therefore, the way of organizing plan of design will be effective in creating quality of influence and penetration.

\subsubsection{VisualPermeability}

It takes place with a continuous space and open design and formal penetration by focus on distinction of space in hall or entrance. Increase the level of activity at edge and appropriate definition for entrance leads to improve influence and penetration [3].

\subsubsection{Legibility}

Lynch argues that driven factors are as effective as fixed factors in creating an image of space in mind in which the foremost concept is legibility. Namely, we can easily identify the spatial elements and relate them within a continuous framework together. Thus legibility may contribute to the individuals to find oneself in space and feel securely [7]. In other words, the person can uniquely recognize the location and form it along with some features like structure, transparency, harmony, and legibility and its concept in observer's mind that has two functions:

- It gives human the power for recognition to read the space.

- It creates the emotional function that is accompanied with sense of security, identity, sense of place, and attachment and generates relation and unity among people and location [16].

\subsection{Personal Space- Territory-Privacy-Traffic and Its Relation with Livability}

As it already mentioned, livability emerges in an interactive platform among environment and human. In this way, it considers social systems for relationships among environment and behavior and in traditional approach the environment is a group of factors, which are related to behavior with causal relationship namely environment affects on human and causes $\mathrm{him} /$ her to show various behaviors. This theory also applies to the cases in which a certain environmental form or frame such as the closed settlements influences in social behaviors of people and also one could deem the environment as consequence of behavior. This can be analyzed from twoaspect attitude based on which it is assumed as effective feature for creating behavior and consequence and effect of 
the given behavior. Regarding design of appropriate environments for people and rate of achievement of designers in prediction of human aspects and implementation of buildings and localities at micro and macro levels, he has drawn their attention toward identifying way of perception, utilization, and valuation of people for environment.

The personal distance is a term that was used by Heidegger [5] to identify the fixed distance among members of noncontact types while in the opposite point, traffic is caused by ever-increasing population of the world and it is deemed as an ecologic disaster, which its first ominous consequence is due to excessive proximity of individuals to each other. In fact, concept of traffic and social isolation is the consequence of individual's failure in access to appropriate level of privacy while the requisite of this territory is where the relations of humans are required both verbal exchange and active use of environment, ownership, and defense.

The four approaches in classification of Allan Westin[24] for privacy include loneliness, proximity and closeness in a small group (wife and husband), anonymity (individual presence in public place lonely), or taciturnity (creating psychological barrier against unwanted intrusion). According to attitude of Proshansky[25], individuality- growth in individual independence interaction- in borderline among individuals, life cycle in several stages of life, difference in character and life of persons, supervision as meaning of freedom of option, asking for help from environment through monitoring over interaction behavior, orientation of duties, actions, and behaviors in nonpublic places and phenomenology based on which privacy is not only a behavioral phenomenon, but also it is a psychological unique experience [18].

Indeed, we are coordinated to this trend with determination of border and in some cases with opening it for nutrition, education, and establishing emotional relationship [19]. Based on the conditions in which the individuals may need to these approaches to have appropriate relations at several time periods any person may exploit from these attitudes as it needs and depending on various culture, origins, insight and states of mind in several communities the rate of these limits and their time interval will be different and these settlements can be acquired in a location under title of home by the aid of five senses in parallel with natural environment and through mutual interaction with human.

Concerning to individual's performance in life and his/ her physical mode and nature that is hidden one can refer to word 'façade' that denotes identifying these levels and for it may occur in functions of architecture. However, it may be occasionally replaced with background and it is a difficult task to maintain this view by carriage of its heavy burden since home is a secure shelter where human likes to lie on his/ her bed on it and to achieve his/ her character [5] or according to statement of Pastalan[17]territory achieves a psychological identity with a place and is converted into a symbol with sense of ownership and formal composition. Similarly, the appropriate and proper isolation of individuals may reduce interruptions and stimulations and instead it exerts this pressure to inside the room and its inhabitants. Of course, we should know that both low- density and high- density regions can be pleasant and way of arrangement in space and environment is effective in this trend since space is essentially capable to become both private and public while none of them could act independently. In fact, these mutual relations give the people power for choice as an essential source for sensational improvement. Since in a society where most of its members are permanently living far from each other either there is no borderline between its members or it could not continue the natural life. In such communities, not only there is no balance, but also the motive, viability, and security will be removed from them.

\subsection{Home}

Based on the unfavorable experience of design of buildings whether residential and non- residential is irrelevant to the environment in today world and this fact is clear for all the people that the result of such designs is only based on personal taste of architect. Even this may be valuable per se, but it may practically be converted into a disharmonious system. In order to deserve to be called as a home, that place has not to be absolutely or permanent settlement and or included our dresses. It is adequate for a building to be harmonious with our internal voice and it should be able to justify our attitude so that we can call it as home [20]. Our love to home emphasizes in this point that our identity is not voluntary. As we need to a house for our physical body, we also need to a home for our soul so that to compensate for our weakness and to improve our spirit; or in other words, our available spaces should be deemed as pleasant interpretations of our souls and survive the important and transient aspects of our personality. The home is deemed as an envelope that establishes relationship among outside environment and biologic phenomena of human. An individual should live along with a family in a home. Namely, s/he should sleep, walk, lie down, see, and think. From viewpoint of Le Corbusier, both aspects of physical and spiritual needs of human will be met with spatial organization of home [21].

Based on Robert Gifford's opinion, six features of a home are as follows:

- It is a haven that has encompassed the person with his/ her privacy, security, safety, and guarding against difficulties of outside life.

- The person may find his/ her position in the world by the aid of home and acquires bravery and find the way to organize the life.

- The home plays role in social identity of human and $\mathrm{s} / \mathrm{he}$ receives sense of relative, attachment, and social position from the home.

- This occurs in the home where the person feels oneself as a member of family, group, and culture.

- Warmth of home is heated with the above-said features but it exceeds from them and becomes symbolic.

- The physical proportion means that form and structure of home should be consistent with mental needs of individuals [22]. 


\section{Conclusion}

Human is a creature that has overcome on environmental conditions and nature of positions in his/ her settlements and has led his/ her life in public and private territories with integration of technology, space, and sensational perception of his/ her everyday life. In this regard, determination of rules for design of several spaces is assumed as paramount measure that provides the appropriate mutual relationship with proper size through keeping the healthy density in treatment and continuance the sense of duration of individual and collective identity among individuals. Creating such principles requires making several efforts and the needed expertise that can operate together and at large scale. Rather than providing shelter for human along with nature, the consistency of human's activities in coordination with the nature in architecture will lead to heath of soul and natural beauty in environment and whereas such environments are adaptable and flexible even though design of them does not provide consistency among human and environment thus it requires deeper review on basis of theories and principles of design.

As the main axis of subject in the world and with respect to his/ her requirements, Human is placed at top point of considerations in research and design. The spatial analysis of behaviors is a heuristic measure in completion of intuitive impressions of designers in order to acquire richer recognition of human's behaviors since several places create difference feelings in various people and subjective background and cultural field of individuals may play effective role in this trend. The studies have shown that in those communities where more time is spent for important human relationships they witness lesser stress and conflict. Design of an appropriate settlement is meant paying attention to need of users and beneficiaries at the same time since most of these requirements are unconscious and often people could not recognize and organize them properly and in environments where human's perception can be improved along with happiness. In other words, the formal, functional, and intellectual features of the place may affect on formation of cognitive, emotional, and behavioralinteraction with the place as some themes in psychological concepts of design. In this regard, this means all those points, which are perceived by the senses. With creating concepts and providing certain activities in generating sense of place, the formal features may affect on spaces and these activities mean the functional aspects of place in meeting human's requirements. Similarly, term concept here means a property of a phenomenon that relates it to conceptual and aesthetic aspects of life and after perceiving the environment by the given person it can decode those concepts and meanings.

Table 1. The effective elements on livability of territories and domains [23]

\begin{tabular}{ll}
\hline & Flexibility and variability \\
& Proper traffic mixing \\
Environmental & Paying attention to green space and furniture \\
& Paying attention to quality and lighting of environment \\
& Paying attention to perception of colour in the face of spaces \\
& Paying attention to signs, which express distinctive features from that urban environment \\
Cultural & Survival and respect for environment \\
\hline
\end{tabular}

\subsection{Presentation of Strategies in Environmental Design}

Overall and with respect to what it implied, the criteria for livability in environmental design can be classified in four groups, which are the formal criteria including physical strategies in design and furniture; sensational criteria comprise of light, color, and psychological measures in environment; performance (functional) criteria such as paying attention to activities day and night; and cultural criteria like paying attention to cultural requirements and values of users from the space as they have been summarized in the following table. These factors contribute to design strategies to act more definitely and scientifically act in process of education of environmental design.

Table 2. Strategies in environmental design

\begin{tabular}{|c|c|c|}
\hline \multirow{9}{*}{ 1- Formal } & \multicolumn{2}{|c|}{ Restriction of space Continuity of space with environment and scale as a continuous system } \\
\hline & \multicolumn{2}{|c|}{ Creating spatial distinction by the aid of pavement and lighting especially in space for old persons and children's play } \\
\hline & \multicolumn{2}{|c|}{ Legibility by means of plants with observance of certain principles } \\
\hline & \multicolumn{2}{|c|}{ Start and end i.e. using element at the beginning and end of area } \\
\hline & \multicolumn{2}{|c|}{ Accessibility with creating various ways of access } \\
\hline & \multicolumn{2}{|c|}{ Increase in number of entrances surrounding general edges } \\
\hline & \multicolumn{2}{|c|}{ Paying attention to various buildings as indentation or perturbation in façade } \\
\hline & \multicolumn{2}{|c|}{ Paying attention to design of gates, sidewalks, and urban furniture } \\
\hline & \multicolumn{2}{|c|}{ Accessibility with creating various paths and rising public transportation for passage } \\
\hline \multirow{5}{*}{ 2- Sensational } & \multirow{5}{*}{$\begin{array}{l}\text { Increase in sensational relation and } \\
\text { treatment with environment }\end{array}$} & Creation of outdoor open space and link with nature \\
\hline & & Creating rich mental perception and image and various experiences \\
\hline & & Visual and functional duration \\
\hline & & Creating new buildings with observance of sky line and floor \\
\hline & & Creating attractiveness through main routes for stability \\
\hline
\end{tabular}




\begin{tabular}{ll}
\hline Colour & \multicolumn{1}{c}{$\begin{array}{l}\text { Using colours, boards, and inspiring pictures at entrance, lobby or furniture } \\
\text { Balance or harmony (using of stronger colour of the same family) }\end{array}$} \\
Using of deep colours and emphasis on rhythm particularly at line of demarcation among spaces \\
$\begin{array}{l}\text { Lighting (creating of perspective by the aid of light adjustment) } \\
\text { Creating self-sufficient spaces }\end{array}$ \\
$\begin{array}{l}\text { Principal separation of automobile and human } \\
\text { composition proportion, and scale } \\
\text { maximization of ease of use }\end{array}$ \\
$\begin{array}{l}\text { Paying attention to zoning } \\
\text { Providing opportunities for remaining alone in the public }\end{array}$ \\
$\begin{array}{l}\text { Creating round-the-clock activities by the aid of opening shop, exhibition, 24-hour library or multi-purpose halls } \\
\text { Paying attention to both public Allocation of two front and rear sided to public and private areas respectively } \\
\text { and private aspects of space } \quad \text { Transparency with creating permeable level between public and private spaces }\end{array}$ \\
$\begin{array}{l}\text { Rising permeability by the aid of construction of smaller and more popular blocks } \\
\text { Creation and building of compatible spaces to requirements and models of public adjacency } \\
\text { Creating link with classic architecture and paying attention to old buildings }\end{array}$ \\
Paying attention to sedentary life, walking, bicycle- running and healthy lifestyle, slowly running, and skate roller etc \\
Creating public spaces based on wide range of activities including fairs, performances, and plays \\
Privatization
\end{tabular}

\section{References}

[1] S.Haim, "English-Persian dictionary Haim", 4th edition, Tehran, 2002.

[2] J.Lang, "Creating architectural Theory", University of Tehran press. 2557,5 th edition. 2009

[3] I. Bentley, Alcock, Murrain, M cGlynn and smit." Responsive Environments, A manual for designers", 2006

[4] www.anthropology.ir

[5] E.Hall,'The hidden dimension", University of Tehran press. 2355, 6th edition.2005

[6] Saeedi, sheshpari,"Urban reflection on the concept of meeting space. Staying in space", News magazine Architecture Utopia, no.36, 2011.

[7] K. Lynch, "The Image of the City",Cambridge Massachussettes, MIT Press 1960.

[8] K.Calman, "Quality of life in cancer patients",ahypothesis. Journal of Medical Ethics, 10, 1994, 124-128.

[9] C.Ferrance, M.Powers" Quality of life index: development and propertiedAdvnursci1985

[10] M.Testa\&D.Simonson." Assessment ofquality-of-life outcomes" New England Journal of Medicine, 1996, 334, 835-840.

[11] E.Nel,"The effect of preparative Teaching on the emotional attitude of patients undergoing coron, 1993.

[12] S.Behboodian," promoting new vitality in public spaces by organizing a human-centered approach”, MSc Thesis, University of Tehran, 2011

[13] M.Moudy",The Application of color in ergonomics, Tehran, 2000

[14] K.Mahmoodi, A.Shakibamanesh,"Principles of color in architecture and urbanism "Tehran, 2005

[15] Gh.HagigatNaiini,"Essay and Its Impact on Urban Painting" Art Quarterly Bulletin", No 3,2001

[16] N.Toulayi," New forms of solidarity", Iran, Amir Kabir Press, 2007

[17] J.Pakzad." Manual analysis of urban spaces",ShahidiPress, Third edition.2007

[18] I.Altman,"The environment and social behavior",second edition, 2003

[19] A.Simmel,"Privacy is not an isolated freedom". New York, Atherton press, 1971

[20] A.de Botton," Architecture of Happiness", First Edition,2009

[21] M.R.Haeri, "contemporary house-features and needs", Abadi Journal Issue 28, 2003

[22] R.Gifford, Psychology residential environments, translating vahidQobadian, Journal of Architecture and Culture, No.2, 3.1999 .

[23] S.Bitaraf, Gh.Keramati,"Evaluate the impact of public spaces, residential townships organizational vitality of the resident", The National Conference on Urban Planning and Architecture in time, International University of Qazvin, Iran, 2014.

[24] A.Westin, Privacy and Freedom; Atheneum, NewYork. 1970.

[25] H. M.Proshansky, W. H., Ittelson, L. G.Rivlin, Environmental psychology: People and their physical settings; Holt, Rinehart \& Winston; New York. 1976. 